\title{
Memórias e reflexões de um professor brasileiro de controle de sistemas dinâmicos
}

Celso Pascoli BOTTURA, Laboratório de Controle e Sistemas Inteligentes, Faculdade de Engenharia Elétrica e de Computação, UNICAMP - Universidade Estadual de Campinas, 13083-852 Campinas, SP, Brasil.

\begin{abstract}
Resumo. Em 1961 começou a Pós-Graduação no Brasil. Nestes 50 anos o sucesso e o alcance desta iniciativa e sua influência sobre as Universidades brasileiras, tanto no ensino como na pesquisa são formidáveis. Inicialmente o Mestrado foi priorizado. Em 1969, o Doutorado começou de forma quase que artesanal, dada a inexperiência em pesquisa no Brasil, e foi crescendo gradualmente. Nos últimos 20 anos ocorreu um processo bem sucedido de amadurecimento e crescimento e hoje podemos dizer que temos pesquisadores e programas maduros de Pós-Graduação nas mais variadas áreas e universidades brasileiras, embora a nossa ciência e a nossa engenharia ainda estejam bem distantes do que os melhores centros mundiais realizam. Paralelamente e por causa de tal processo, várias sociedades científicas e/ou congressos foram sendo criados e consolidados. Neste trabalho apresento algumas memórias e reflexões sobre este processo com ênfase na minha área de atuação como Professor e Pesquisador: Controle de Sistemas Dinâmicos e na minha vivência.
\end{abstract}

Palavras-chave. Controle, sistemas dinâmicos, história.

\section{Introdução}

Recentemente publicamos na Revista Controle e Automação da SBA-Sociedade Brasileira de Automática, o artigo [10]. Ele foi motivado por um convite à SBA pelos organizadores do "2009 IEEE Conference on the History of Technical Societies", um evento do 'Institute of Electrical and Electronics Engineers-IEEE" dos Estados Unidos da América, com o propósito de explorar a história de sociedades técnicas: científicas e tecnológicas ao redor do mundo e de celebrar os 125 anos de criação do IEEE em Filadélfia em 1884.

Depois de interagir com vários membros da SBA, o seu então Presidente, Carlos Eduardo Pereira, da UFRGS e eu escrevemos um trabalho [29] sobre a história da SBA para aquele congresso. Em agosto de 2009 apresentamos tal trabalho. Tal participação memorável permitiu-nos interagir com muitos Presidentes e membros atuais, bem como com mais antigos, do IEEE e de Sociedades Científicas tradicionais de vários Países: Alemanha, França, Inglaterra, Japão, China, Rússia,..., bem como de outras americanas como a American Society of Mechanical Engineers-ASME, a American Society of Civil Engineers-ASCE,...

A importância que o IEEE dá a sua própria história, bem como a importância que os americanos dão na história americana às atividades técnicas e às pessoas 
que as exercem e desenvolvem são notáveis. Isto me faz lembrar e ressaltar uma experiência que vivi ao visitar o Museu de História Americana em Washington, em 1991. Pensei que veria, como provavelmente seria o caso em nosso e outros países, em um museu de história, políticos e guerreiros. Para minha surpresa e satisfação vi algo inesquecível, emocionante e muito importante: a importância que os americanos dão à Tecnologia e à Ciência em sua história; ficaram na minha memória uma figura enorme e excepcionalmente bem posicionada de Thomas Alva Edison e diversas máquinas, equipamentos e dispositivos que eles desenvolveram e que revolucionaram a vida humana.

Este artigo foi motivado pelo convite que recebi da SBA e dos organizadores do "DINCON 2011 - 10 ${ }^{a}$ Conferência Brasileira de Dinâmica, Controle e Aplicações" , para realizar sua Palestra de Abertura. O tema deste congresso: Dinâmica e Controle, visto de forma multidisciplinar é muito importante e significativo para mim. O DINCON 2011 ganhou nova estrutura e passou a ser organizado por múltiplas sociedades científicas brasileiras envolvidas com Dinâmica e Controle:

- ABCM: Associação Brasileira de Ciências Mecânicas,

- SBA: Sociedade Brasileira de Automática,

- SBMAC: Sociedade Brasileira de Matemática Aplicada e Computacional.

Neste artigo abordo alguns aspectos que chamaram a minha atenção, inicialmente como aluno do ITA, e em meus 50 anos de vida profissional, e que gostaria de ressaltar e compartilhar com a comunidade de matemática aplicada e computacional, em particular com a de dinâmica, controle e automação.

\section{Educação Superior No Brasil: Algumas Consi- derações}

A educação superior no Brasil começou há relativamente pouco tempo, mesmo em relação à nossa curta história, e à própria curta história da educação superior na história da humanidade. Por razões culturais e políticas, a Educação Superior no Brasil começou muito lentamente, essencialmente depois que Napoleão forçou a mudança do Rei de Portugal para o Brasil em 1808. Nos Estados Unidos e em algumas colônias espanholas da América Latina a Educação Superior começou há mais de três séculos.

- As primeiras universidades brasileiras foram criadas apenas no século 20, com uma forte influência européia.

- Atividades agrícolas forçaram algum desenvolvimento tecnológico no século passado. A partir de 1930, as atividades industriais ganharam maior impulso. A partir da década de 1950, especialmente a partir de 1956, a indústria automobilística começou a produzir tratores e carros no Brasil . A produção de caminhões começou um pouco antes.Anteriormente, mesmo bicicletas e ferramentas, eram importadas. 
- A Segunda Guerra Mundial provocou novas e importantes mudanças na cultura e nas instituições brasileiras.Uma muito importante para a Educação Superior brasileira foi a criação do Instituto Tecnológico de Aeronáutica-ITA, uma instituição de Ensino Superior, dentro do ex-Ministério da Aeronáutica, porém fora do Ministério da Educação. O ITA é a primeira Instituição de Ensino Superior brasileira puramente inspirada nas instituições de Ensino Superior americanas, particularmente no MIT - Massachusetts Institute of Technology. O ITA começou em São José dos Campos, SP, em 1950, como a concretização de uma concepção que Casemiro Montenegro Filho[27,30 ], um oficial militar brasileiro da Aeronáutica criou ao visitar o MIT, durante a segunda guerra e vislumbrar a sua importância para o que o Brasil deveria fazer em Educação Superior de qualidade e tecnologia. O primeiro Reitor do ITA, o americano Richard H. Smith (1946-1951), era Professor do MIT, e produziu o famoso "Plano Smith"para o ITA. Os próximos três Reitores do ITA também foram americanos. O ITA, a partir de 1961, foi a primeira instituição educacional brasileira a adotar integralmente, o modelo de educação de Pós-Graduação das universidades americanas, com base em créditos em disciplinas, mestrado e doutorado.

A partir de então, o paradigma de Educação Superior no Brasil mudou completamente, e gradualmente passou a seguir o modelo do ITA.

- A primeira comissão de Pós-graduação do ITA foi constituída pelos Professores Doutores:

- Alexander Allen, americano, seu Presidente,

- Jacek P. Gorecki, polonês,

- José T. Senise, brasileiro,

- Luiz V. Boffi, nascido na Itália e

- Padraic C. Dunne, inglês.

- Minha turma, de alunos do ITA, ITA-62, prestou uma homenagem ao primeiro Reitor brasileiro do ITA, o Professor Marco Antonio G. Cecchini, em maio de 2011, e dele recebemos alguns textos por ele reunidos com o título: "O ITA Que Eu Conheci"[13]. Neste há um texto dos então Professores do ITA : José Thomas Senise e Marco Cecchini, "A Criação da Pós-Graduação Pelo Instituto Tecnológico de Aeronáutica", do qual eu extrai as seguintes informações:

"Coube ao ITA, em 1961, oferecer no Brasil, primeiramente, na área de engenharia, o mestrado, segundo o modelo americano, também chamado, entre nós, de pós-graduação stricto sensu. Nesse ano também foi criado o mestrado em agronomia, pela então Escola Superior de Agricultura e Veterinária de Viçosa que, à semelhança do ITA, vinha recebendo a colaboração de professores americanos. Dois anos mais tarde, em 1963, o oferecimento do mestrado a engenheiros recebeu um grande impulso, com a criação da COPPE-Comissão Coordenadora dos Programas de Pós-Graduação em Engenharia, mantida pela 
então Universidade do Brasil. O Prof. Alberto Luiz Galvão Coimbra, que viria a se tornar Coordenador da COPPE, visitara o ITA em 1961 e demonstrara interesse em aproveitar a Experiência do Instituto para estruturar o mestrado em engenharia química, especialidade pela qual começaria a pós-graduação dessa Coordenação. Ele confirmou esse interesse em carta dirigida a um de nós(Senise) em 1962. Não há dúvida de que a pós-graduação oferecida pelas três instituições universitárias acima citadas serviu de modelo para que o conselheiro Newton Sucupira estruturasse o seu importante Parecer que, sob número 977, seria aprovado pelo Conselho Federal de Educação, em 03 de dezembro de 1965. Nesse Parecer é ressaltado o mérito do modelo americano de pós-graduação, adotado pelas três instituições universitárias.A pós-graduação de todos os campos do conhecimento oferecida hoje por todas as instituições universitárias brasileiras segue o Parecer 977. É interessante assinalar que o modelo americano de pós-graduação, iniciado e adaptado por escolas de engenharia brasileiras, com relativamente pouca experiência nessa modalidade de ensino, tenha conseguido alterar o ensino pós-graduado de campos do conhecimento, como o das ciências, das letras e do direito, onde esse tipo de ensino vinha sendo desenvolvido desde a década de 1930."

- O ITA deu origem a instituições de grande importância para o Brasil: EMBRAER, INPE...

\section{Educação em Controle Automático no Brasil: Iní- cios}

Em 1953, o americano Edward W. Kimbark, PhD pelo MIT e primeiro diretor da Engenharia Eletrônica do ITA, lecionou o primeiro curso de controle automático em uma escola de engenharia brasileira. A partir de 1954, Otto V. M. Smith, professor da University of California, Berkeley, passou alguns anos ensinando controle automático no ITA. Em 1957, o professor Luiz Valente Boffi, retornou ao ITA com um doutorado pelo MIT e seria o primeiro grande líder da educação em controle automático no Brasil.O professor Boffi organizou o primeiro grupo para educação e pesquisa em controle automático do Brasil no Departamento de Engenharia Eletrônica do ITA. Um dos membros de tal grupo era Manoel Sobral Jr.. Ele formou-se no ITA em 1958 e realizou pós-graduação na University of llinois, onde obteve o $\mathrm{PhD}$ em 1964. O professor Manoel Sobral lecionou o primeiro curso de controle automático na UNICAMP em 1970 e realizou um trabalho excepcional como o primeiro grande diretor da então Faculdade de Engenharia de Campinas da UNICAMP. Ele criou o primeiro Departamento de Automação em uma universidade brasileira, cujo primeiro chefe foi o professor Yaro Burian Jr., que também veio do ITA em 1971. O Departamento de Automação da UNICAMP teve nos seus primeiros anos 28 membros, dos quais 15 vieram do ITA e 10 da UNICAMP. Dentre seus membros cito: Celso Pascoli Bottura, Hermano de Medeiros Ferreira Tavares, Marcio Andrade Netto, José Cláudio Geromel, Ernesto Ruppert Filho, Manuel de Jesus Mendes, Mário Jino, Luiz Gimeno Latre, Alvaro Badan Palhares, Wagner Caradori 
do Amaral, Maria Adelia Collier Farias, Wang Binseng, José Wilson Bassani, Leo Pini Magalhães, Clésio Luiz Tozzi, Akebo Yamakami e Secundino Soares.

Na Universidade do Brasil, em 1963, hoje Universidade Federal do Rio de Janeiro-UFRJ, foi criada a COPPE-Coordenaçãodos Programas de Pós-Graduação em Engenharia, com características especiais devidas às dificuldades culturais e estruturais daquela época para naturalmente criar cursos de pós-graduação em universidades brasileiras, diferentemente do que passou a ocorrer posteriormente. Hoje que a pós-graduação baseada no modelo americano é um grande e respeitável sucesso no Brasil é difícil acreditar em certas dificuldades iniciais, que de fato ocorreram. As peculiaridades do ITA e da COPPE-UFRJ foram muito importantes para tal sucesso. Na América Latina, com a possível exceção do México, e talvez do Chile, podemos dizer que até hoje este fenômeno educacional ainda está para acontecer e ter o impacto que a iniciativa do ITA teve na educação moderna brasileira. A COPPE recebeu um impulso muito forte do Governo brasileiro, que então começara a dar maior atenção à pós-graduação, particularmente em engenharia. Eles começavam a perceber a importância que as iniciativas do ITA nesta direção tinham para a educação e o desenvolvimento do Brasil, em especial para o Ensino Superior brasileiro de qualidade. A COPPE colocou muita ênfase na educação pós-graduada em engenharia, em especial nas áreas de Controle Automático, Pesquisa Operacional, Energia e Computação. Dentre seus professores cito: Pravin Varaya, Shankar P. Bhattacharyya, Carlos Juarez Távora, Julio Coutinho, Liu Hsu, João Lizardo de Araujo, Clovis Gonzaga, Afonso Celso Del Nero Gomes, Eugenius Kaszkurewicz, Richard Magdalena Stephan, Edson Watanabe, Luiz Pereira Caloba, Sandoval Carneiro Jr, Nelson Maculan Filho, Jacques Szczupak, Alkindar Pedroso, Ronaldo Marinho Persiano, Luiz Pinguelli Rosa e Adilson de Oliveira.

Na Escola Politécnica da USP os primeiros cursos de controle automático foram lecionados pelos professores Plínio B. L. Castrucci e Luis A. G. C. de Barros Barreto, em 1960. O segundo fora auxiliar do Prof. Kimbark no curso de 1953 do ITA. O professor Castrucci lideraria o desenvolvimento da área de automática da USP, principalmente a partir da década de 1970, quando a pós-graduação passaria a ter um maior desenvolvimento e aceitação nas universidades brasileiras. Assim, a partir da década de 1970:

- Na Universidade Federal de Santa Catarina-UFSC, Walter Celso de Lima lideraria um grupo de automática e de engenharia biomédica, que muito cresceria.

- O mesmo ocorreria na área de automática da Universidade Federal de Campina Grande, então parte da Universidade Federal da Paraíba, liderado por Edson Cabral da Silva, que deu grande ênfase à área de eletrônica de potência.

- Na UFMG liderado por Ronaldo Tadeu Pena.

- Na PUC-RJ liderado por Pedro Magalhães Guimarães Ferreira.

- Na UFPA liderado por Jurandyr Garcez.

- Na UFES liderado por Edson de Paula Ferreira.

TEMA Tend. Mat. Apl. Comput., 14, No. 1 (2013), 23-42.

doi: 10.5540/tema.2013.014.01.0023 
- Na UNICAMP, que começara efetivamente em 1966, a pós-graduação em engenharia elétrica começa em 1972, com grande ênfase em controle e automação e em telecomunicações. Nos beneficiamos de:

- Reforma universitária recente que eliminava a cátedra e criava os departamentos e o tempo integral, dentre outras conquistas, que anteriormente só existiam no ITA.

- O ITA formara um grande número de doutores no exterior, mas sofrera uma grande crise, sobretudo em 1965. O ITA enfrentava problemas estruturais graves. Este pessoal retornara para o exterior, mas estava ávido para voltar e trabalhar no Brasil. Campinas e sua universidade estadual em implantação eram muito atraentes.

- A UNICAMP tinha um Reitor excepcional: Zeferino Vaz[20].

- O Brasil estava em época de milagre econômico.

- A pós -graduação e a pesquisa começavam a ser importantes no Ensino Superior brasileiro.

- No ITA, após a saída do Prof. Luiz Valente Boffi, que se radicou nos Estados Unidos, na University of Michigan, após a grande crise do ITA, o seu grupo de automática na engenharia eletrônica foi liderado por Wladimir Borgest e mais recentemente por Takashi Yoneyama.

- Quatro dos meus colegas da turma ITA-62 que se formaram em engenharia eletrônica passaram toda ou grande parte de suas vidas profissionais na área de automação industrial e controle e se tornaram profissionais importantes nesta área no Brasil: Toshiwo Yoshikai, Maurício Kurcgant, Simão Copeliovitch e Claudio Augusto Câmara.

\section{Algumas Lembranças Pessoais}

Como aluno do quarto ano de Engenharia Aeronáutica do ITA, em 1961, percebi que os meus colegas de Engenharia Eletrônica recebiam uma educação excelente em Análise e Controle Automático de Sistemas Dinâmicos por parte do Prof. Luiz Valente Boffi e seu grupo, e que nos não estudavamos Controle em Engenharia Aeronáutica. Fascinado por Dinâmica de Sistemas e seu Controle, percebi que naquela época, no Brasil, a maioria dos cursos de análise e de projeto em engenharia tinha uma visão ESTÁTICA. Apenas os alunos de engenharia eletrônica ganhavam esta visão DINÂMICA, graças aos cursos de CONTROLE. Através daquela pequena fresta percebi que o que eu procurava chamava-se CONTROLE. Fascinavam-me a DINÂMICA e o CONTROLE DE AERONAVES e de SISTEMAS de uma forma geral.

Para minha sorte, e graças ao engenheiro aeronáutico Francisco Galvão, turma ITA-1959, realizei o Trabalho de Graduação ( na época Trabalho Individual-TI no ITA): "Simulador para Estudo da Estabilidade Dinâmica Longitudinal de Aviões 
Utilizando Computador Analógico", sob orientação do Professor Jacek Piotr Gorecki, $\mathrm{PhD}$ pela Caltech-California Institute of Technology [3,21].

No ITA fiz a opção por cursos na área de Projeto de Aeronaves, na época chamada Técnica Aeronáutica.Éramos apenas três alunos nesta especialidade: Ozires Silva, que seria o grande responsável pela criação da EMBRAER, Antonio Bakowski, que se tornou um grande especialista em Homologação de Aeronaves e eu. Tivemos três excelentes cursos de Projeto de Aeronaves; o primeiro com o Professor americano Clarence Flora, e os demais com o belga Renée Marie Vandaelle.

No final de 1962, quando me formava engenheiro aeronáutico pelo ITA, meu orientador de TI, Prof. Jacek, ofereceu-me uma bolsa de 18 meses para realizar o mestrado em aerodinâmica nos Estados Unidos. No Brasil de 1962, mesmo no ITA, nós nunca ouvíramos falar em Mestrado, a menos de poucas exceções, nas quais eu não me incluía:

- Não sabíamos o que era Pós-Graduação, nem Mestrado. Disse ao Prof. Jacek que se ele me explicasse o que eram Pós-graduação e Mestrado e me conseguisse um estágio em fábrica americana de aviões, eu aceitaria sua oferta. A oferta de trabalho que mais me interessava na época era a da Companhia Aeronáutica Neiva, em Botucatu, São Paulo, onde eu estagiara por 2 meses no início de 1962. Eles queriam me contratar e a recíproca também era verdadeira. O Prof. Jacek paciente e gentilmente explicou-me o que eram a pós-graduação e o mestrado e disse-me que a primeira defesa de Mestrado em Engenharia no Brasil ocorreria no início de 1963, no ITA!

- Mesmo sendo aluno de graduação do ITA, desconhecíamos isto que estava começando no Brasil, no ITA!

- Atualmente no Brasil, onde a pós-graduação é um grande e respeitável sucesso, é aceita pelo sistema universitário brasileiro, tem grande importância e é bem conhecida de forma geral, é difícil imaginar o que eu descrevi acima sobre a minha experiência pessoal de 1962. Mas as coisas eram assim.

- O Brasil sofreu uma grande transformação educacional em todos os níveis. Apesar de todos os problemas e dificuldades, apesar de todas as críticas e autocríticas, nossa educação melhorou mais do que piorou. A educação superior, a partir da década de 1960 ficou cada vez melhor e para isso o modelo americano foi a principal influência positiva e inclusiva em todos os níveis, sobretudo depois da Reforma Universitária em fins da década de 1960. Ainda há muito a melhorar, mas o salto foi enorme. Aceito pela Purdue University, completei meu mestrado na sua School of Mechanical Engineering, em 1964.

- O grupo de Controle Automático da Engenharia Mecânica da Purdue University era liderado pelo Professor Rufus Oldenburger. Em 1964, os Profs. C. Rezek e R. Goodson, deste grupo, lecionaram pela primeira vez o curso "Instrumentation for Dynamic Systems"e felizmente eu fui aluno deste curso e informei o Prof. Cecchini, então reitor do ITA, sobre isto. Após realizar estágio na fábrica de Aviões da Cessna Aircraft Company, Wichita, Kansas, 
retornei ao ITA no início de 1965.O ITA me deu a grande oportunidade para criar o Grupo de Instrumentação e Controle da Engenharia Mecânica no ITA.

- Em 1965, eu comecei a lecionar cursos de graduação e de pós-graduação em Controle Automático e Instrumentação para Sistemas Dinâmicos para alunos de engenharia mecânica e de engenharia aeronáutica, pela primeira vez no Brasil, no ITA.

- Para fazer isto eu recebi uma contribuição muito importante da University of Michigan, que ajudava o ITA a implantar seu curso de Engenharia Mecânica, cuja primeira turma se formaria em 1965.

Para realizar este importante trabalho quero registrar as importantes contribuições de:

- Jerome Hemmye, professor americano da University of Michigan, que como professor do ITA, antes de voltar para os Estados Unidos em 1965, me abriu as portas para que eu realizasse um dos mais importantes trabalhos da minha vida profissional: criar o Grupo de Instrumentação e Controle do Departamento de Projetos da Divisão de Engenharia Mecânica do ITA de 1965 a 1970.

- Prof. Raymond G. Jacquot, americano, que como aluno de doutorado na Purdue University lecionou-me um curso excepcional de Vibrações Mecânicas. Posteriormente ele escreveu um excelente livro didático de controle digital [23].

- Prof. João Hernandes, que em 1959, em um curso de Dinâmica no ITA, foi um grande mestre de Vibrações Mecânicas.

- Profs. Sergio Nello Vannucci, Claudio Nobuo Takase e Antonio Luiz Sansão, meus ex-alunos no ITA, que me ajudaram a criar o referido grupo e realizaram trabalhos essenciais.

- Atualmente aquele Grupo de Instrumentação e Controle é o de Mecatrônica do ITA e é liderado pelo Prof. Luiz Carlos Sandoval Góes.

- Em 1969, o Prof. Fernando Pessôa Rebello do ITA foi convidado pelo Reitor da UNICAMP, Zeferino Vaz, para coordenar a implantação da engenharia mecânica da sua então Faculdade de Engenharia de Campinas-FEC.

Empolgado com o nosso trabalho no ITA, o Prof. Rebello convidou-me para implantar na engenharia mecânica da UNICAMP um Grupo de Instrumentação e Controle nos moldes do que eu estava realizando no ITA. A UNICAMP era uma universidade muito promissora, mas ainda com quase tudo por fazer, inclusive sua infra-estrutura. A engenharia, por exemplo, estava precariamente instalada no que viria a ser seu Colégio Técnico COTUCA. Fiz o projeto em 1969, para tentar criar e viabilizar em 1970, o Grupo de Instrumentação e Controle da Engenharia Mecânica da UNICAMP, para o qual era essencial, como eu o fizera no ITA, instalar um Laboratório de Instrumentação e Controle, para o qual especifiquei os equipamentos a serem comprados. 
Dificuldades de várias ordens ocorreram e outros acontecimentos e prioridades se interpuseram. Porém meu projeto foi entregue ao meu coordenador Prof. Rebello e ao primeiro diretor da FEC, Professor José Fonseca Valverde, em 1969 . Em 1970 entreguei o meu projeto ao segundo diretor da FEC: Theodureto Souto, que substituiu o Prof. Valverde, após algumas dificuldades institucionais [20].

Em 1970, graças ao Professor Theo Guenter Kieckbusch, então da Engenharia de Alimentos da UNICAMP, aproveitamos uma disciplina de Instalações Industriais já existente, para criar uma disciplina de Instrumentação e Controle e Instalações Industriais, e através desta solução lecionei Instrumentação e Controle para a primeira turma de Engenharia de Alimentos da UNICAMP, que se formaria em 1971.

Ainda em 1970, o Prof. Manoel Sobral Junior, que viria a ser o terceiro diretor da FEC, tomou conhecimento do meu projeto para a engenharia mecânica da UNICAMP e de certas dificuldades que independiam da minha vontade, para então realizar o mesmo. O Prof. Sobral não esqueceu meu plano nem o sonho do Prof. Rebello de que os alunos de engenharia mecânica da UNICAMP tivessem cursos de Instrumentação e Controle. Contudo, havia muita coisa por fazer, e outras prioridades e dificuldades se impunham.

Em 1971, quando a FEC passaria a ter efetivamente um corpo de professores em tempo integral, brilhantemente liderados pelo Prof. Sobral, este me convidou para trabalhar em tempo integral na engenharia elétrica da UNICAMP e eu felizmente aceitei. O Prof. Sobral, que conhecia o meu projeto acima comentado, encarregou-me da chefia do Laboratório de Automação da UNICAMP, que passei a implantar imediatamente. A UNICAMP tinha um Reitor excepcional, estava em fase de implantação, e em pouco tempo criamos uma infra-estrutura de ensino e pesquisa de qualidade em automação e controle a partir de quase nada. Priorizei o meu doutorado que começara no ITA em 1969 e sob orientação do Prof. Yaro Burian Jr. tornei-me Doutor em Engenharia Elétrica pela UNICAMP em 1973. Na minha tese de doutorado, as contribuições importantes foram em caos e seu controle em controle de motores serie de corrente contínua por recortador("chopper"). A palavra caos ainda não era usada como passou a sê-lo posteriormente. Nós o chamávamos de oscilações pseudo-aleatórias ou fenômeno de Pulkin-Birkhoff, por sugestão do meu orientador [4]. Fomos influenciados por um projeto de pesquisa que conseguimos com a Companhia do Metropolitano de São Paulo, que estava construindo o primeiro sistema metroviário do Brasil, que seria inaugurado em 1974 [31]. A Companhia do Metropolitano de São Paulo usava a mesma tecnologia que a Westinghouse estava desenvolvendo para o metropolitano de San Francisco nos Estados Unidos, também em construção. Durante nosso projeto percebemos que a Westinghouse limitava a largura dos pulsos do sistema de controle da máquina elétrica por largura de pulso, provavelmente por razões empíricas; por exemplo, para evitar problemas de comunicação, donde de controle. Este resultado empírico foi obtido teoricamente por nós na minha tese de doutorado, ao modelar o sistema através de recorrências 
não lineares implícitas, explicitar a existência de oscilações caóticas e projetar sistema de controle com modulação em largura de pulsos evitando oscilações sub-harmônicas e oscilações caóticas.

Em 1973, no Segundo Congresso De Engenharia Mecânica, no Rio de Janeiro, apresentamos o trabalho: "Controle por Comutação de Motor Série", com alguns dos resultados da minha tese de doutorado [12]. Na mesma seção houve a apresentação de um trabalho do Prof. Hans IngoWeber. Nós o conhecemos e em 1974 o Prof. Manoel Sobral Junior o contratou para trabalhar na engenharia mecânica da UNICAMP, para que ele, como especialista em Dinâmica liderasse a Área de Projeto Mecânico e inclusive provocasse pesquisas em controle na engenharia mecânica e de certa forma ajudasse-nos a dar continuidade àquele projeto inicial de 1969 de dar formação em controle aos alunos de engenharia mecânica da UNICAMP, conforme meu projeto motivado pelos planos e sonhos do Prof. Rebello. Utilizaríamos essencialmente os cursos de controle e os professores da engenharia elétrica para os alunos de engenharia mecânica da UNICAMP que tivessem tal interesse. Colaborei com este projeto lecionando em 1974 a primeira disciplina do mesmo: "Análise Dinâmica de Sistemas". Fui auxiliado pelo Prof. Ernesto Ruppert Filho da Engenharia Elétrica. Um detalhe curioso e importante me ocorre:

- Não estavam previstas aulas de laboratório para esta primeira turma, pois mais professores seriam necessários. Porém, professores e alunos da engenharia mecânica desejavam enfaticamente que houvesse estas aulas. Sensibilizado por tais aspirações, conversei com o meu aluno de então das disciplinas "Análise linear de Sistemas"e "Princípios de Controle e Servomecanismos"[5,6,19], ambas com aulas de laboratório para os alunos de engenharia elétrica da UNICAMP, José Claudio Geromel. Este, como sempre, me surpreendeu positivamente: não só aceitou dar as aulas gratuitamente, como preparou-as primorosamente e trouxe colegas para ajudá-lo. Desta forma, aqueles primeiros alunos dos cursos de controle da engenharia mecânica da UNICAMP tiveram um excelente primeiro curso de Análise Dinâmica de Sistemas. O professor José Cláudio Geromel tornou-se um dos principais professores e pesquisadores em controle de sistemas dinâmicos, com diversos livros publicados na área, no Brasil e no exterior $[14,17,18]$.

Depois disto,o grupo de projeto mecânico da mecânica cresceu bastante sob a liderança do Prof. Hans Ingo Weber e eu que sucedera o Prof. Yaro Burian Jr. como chefe do departamento de automação da engenharia elétrica da UNICAMP, tinha novas prioridades em dinâmica, controle e otimização, pois nossa universidade estava em franco desenvolvimento para tornar-se uma grande universidade brasileira, com grande ênfase em Pesquisa e Pós-graduação.

Em 1980, na primeira tese de doutorado que orientei:

- "Controle por Realimentação de Estado e Modulação em Largura de Pulso de um Servoposicionador Eletrohidráulico: Análise das Oscilações"de Álvaro Geraldo Badan Palhares, além da originalidade na análise e controle de dinâmica 
caótica por realimentação de estado, sua defesa de tese teve uma particularidade que gostaria de destacar:

- "Durante a defesa, a banca foi conduzida para o nosso laboratório de pesquisa e teve a oportunidade de ver experimentalmente que os resultados experimentais e os calculados para a existência de oscilações caóticas coincidiam. Não só produzimos oscilações caóticas para aquele sistema não linear complexo e real, como as controlamos perante a banca, conforme o projeto do controle previamente desenvolvido com base na teoria. Qualitativa e quantitativamente os resultados originais foram excelentes"[28].

\section{Sobre a Fundação de Algumas Sociedades Cien- tíficas Brasileiras}

Com a criação dos cursos de pós-graduação, inicialmente, por uma questão de bom senso e de realismo, enfatizando o Mestrado, já que os números de pesquisas, de pesquisadores experientes, de alunos de pós-graduação, de recursos financeiros e administrativos, e de instituições de ensino superior de nível adequado eram incipientes, foram sendo criadas as condições para:

- Criação de Congressos Científicos com participação brasileira de melhor nível

- Criação de Sociedades Científicas brasileiras nas mais variadas áreas.

- Criação de Programas de Doutorado com condições efetivas de alcançar nível adequado.

- Criação de Revistas Científicas brasileiras de bom nível.

Em 1968, no ITA, foi criado sob a liderança do seu Professor Alberto Von Ellenrieder:

- O Congresso Brasileiro de Pesquisa Operacional - CBPO e logo após a Sociedade Brasileira de Pesquisa Operacional - SOBRAPO, cujo primeiro presidente foi o professor da Escola Politécnica da USP: Oswaldo Fadigas Fontes Torres, que fora nosso professor de Estatistica e de Pesquisa Operacional no ITA. Ele era um engenheiro formado na Poli-USP, que como professor do ITA fizera mestrado em Aeronáutica no MIT. Na crise de 1965, ele deixou definitivamente o ITA e dedicou-se em tempo integral à engenharia de produção da Poli-USP, da qual foi diretor.

- Com a saída do Prof. Fadigas, meu colega Mario Rosenthal, da turma ITA-62, que fizera mestrado na Purdue University, em engenharia industrial, assumiu suas funções. Porém a crise do ITA de 1965 e o mercado de trabalho fizeramno mudar de emprego; mas ele teve a habilidade de trazer para o ITA dois professores argentinos: Alberto von Ellenrieder e Siegfrido Mazza, que fizeram um belo trabalho no ITA, por muitos anos.

- Participei da fundação da SOBRAPO.

- Participei dos primeiros CBPOs e esporadicamente de outros. Na palestra de abertura do primeiro CBPO, no ITA, Siegfrido Mazza se perguntou: "O 
que é Pesquisa Operacional?"e respondeu: "Pesquisa Operacional é o que os Pesquisadores Operacionais fazem". Nunca me esqueci disto. Este tipo de definição se aplica a muitas coisas com as quais convivemos e temos dificuldades para definir.

Em 1973 participamos do Segundo Congresso Brasileiro de Engenharia Mecânica no Rio de Janeiro, porém a ABCM-AssociaçãoBrasileira de Ciências Mecânicas, foi fundada na UNICAMP , em 1975, tendo como fundadores : Luiz Bevilacqua da UFRJ, Hans Ingo Weber, Celso Bottura e Yaro Burian Junior, da UNICAMP, Sidney Stuckenbruck e Solly Andy Segenreich, da PUC-RJ, e teve Nelson Back, da UFSC, como seu primeiro presidente, em 1976, depois de uma fase inicial dirigida por Hans Ingo Weber.

- Apenas no Terceiro Congresso Brasileiro de Engenharia Mecânica, também no Rio de Janeiro,do qual participei, foram publicados os Anais do anterior e deste, e entregues aos congressistas quando da sua inscrição. Naqueles primeiros anos não era fácil publicar os Anais e isto foi passando a ser ponto de honra das Comissões Organizadoras de Congressos. Hoje as coisas são diferentes de várias formas, e o papel foi substituído pelo DVD/CD/Pen Drive.

- Ainda participei do Quarto COBEM, em Florianópolis, e esporadicamente de outros, pois me envolveria mais profundamente com a criação da SBASociedade Brasileira de Automática, em 1975.Desta eu tratei no artigo citado no início deste e aqui, além de sugerir a leitura daquele artigo da Revista "SBA-Controle e Automação", ressaltarei alguns de seus pontos na próxima seção.

Não participei das fundações das sociedades científicas que agora citarei, porém tenho comentários pertinentes:

A SBMAC-Sociedade Brasileira de Matemática Aplicada e Computacional, fundada em 1978, teve como um de seus fundadores e seu primeiro presidente o Professor Odelar Leite Linhares. Lembro-me muito bem dele, que foi meu professor de aerodinâmica no ITA em 1960. Além de excelente professor, era uma pessoa da qual todos gostavam. Lembro-me muito bem do ser humano que ele era, do seu doutorado em Cálculo Numérico, parcialmente realizado na Suiça, de ele ter sido Professor da UNICAMP, da USP-São Carlos e da UNESP-São José do Rio Preto [25].

- Tenho participado do DINCON desde 2003, no ITA, quando ele foi organizado por José Manoel Balthazar da UNESP-Rio Claro e por Luiz Carlos Sandoval Goés do ITA.

- Também me lembro da Criação do Curso de Matemática Aplicada e Computacional no IMECC-UNICAMP, liderada pelo Professor Miguel Taube Neto, turma ITA-63. Empenhado em criar e viabilizar este importante e bem sucedido projeto, ele provocava reuniões das quais participei com outros ex-alunos do ITA e então professores da UNICAMP: Hermano Tavares e Eliezer Arantes da Costa, na segunda metade da década de 1970. 
A SBC - Sociedade Brasileira de Computação, criada em 1978, teve como um de seus fundadores e primeiro presidente Cláudio Zamitti Mammana, da USP, que foi meu aluno da segunda turma do curso de controle automático que lecionei em 1966, no curso de engenharia mecânica do ITA. Fui sócio da SBC durante algum tempo e participei de alguns de seus congressos.

A Sociedade Brasileira de Ciências, hoje Academia Brasileira de Ciências - ABC, foi criada em 1916, na Escola Politécnica da Universidade do Brasil, hoje UFRJ. Tenho muita curiosidade sobre ela, porém pouco sei de sua história.

\section{A Fundação e os Primeiros Anos da Sociedade Brasileira de Automática}

A partir da década de 1970 a pós-graduação brasileira passou a uma fase de maior progresso e aceitação.Vários cursos de Doutorado seguindo o modelo americano foram criados, particularmente em engenharia: ITA, COPPE-UFRJ, USP, UNICAMP.

- Em 1974 eu sucedera o Prof. Yaro Burian Jr. na chefia do Departamento de Automação da UNICAMP.

- Eu achava que já existiam condições para a criação de uma sociedade científica brasileira em Automação e Controle.

Naquela época a palavra "informática"já era muito usada para descrever a área de computação no Brasil. Em 1957 a França criara o IRIA-Institute de Recherche in Informatique et Automatique, atualmente INRIA, que eu visitei em setembro de 1974. "Automatica"era a revista científica da "International Federation of Automatic Control-IFAC".

Em janeiro de 1975, procurei o Professor Plínio B. L. Castrucci no Departamento de Engenharia de Eletricidade da Poli-USP, autor do primeiro livro didático de controle automático no Brasil [12, 19]. Ele reunia tanto a experiência de professor universitário como a de um empresário industrial pois criara a AMPLIMAG, uma empresa pioneira e bem conhecida na área de automação, no Brasil de então. Nós consideramos que a palavra AUTOMÁTICA seria a adequada para descrever, em português, a ciência e a engenharia de Controle e Automação e decidimos criar a Sociedade Brasileira de Automática-SBA, o mais cedo possível. Em 5 de junho de 1975, realizamos a Assembléia de Fundação da SBA em São Paulo, na PoliUSP.Nela foram eleitos Plínio Castrucci-Presidente e Celso Bottura-Vice Presidente da Diretoria 1975-1977.

A SBA foi criada como uma sociedade científica de âmbito nacional, com o objetivo primordial de promover a ciência e a tecnologia de controle automático no seu mais amplo sentido, em todos os sistemas, sejam eles físicos, biológicos, sociais, econômicos, no Brasil e para ser um foro importante para pesquisadores, estudantes e profissionais trabalhando nos vários campos de Automação e Controle. A SBA foi criada para ser "the National Member Organization-NMO of IFAC "para o Brasil, 
conforme explicitado em seus estatutos aprovados na sua assembléia de fundação e implementado logo no início de sua existência.

O primeiro Congresso Brasileiro de Automática foi organizado por Plínio Castrucci, na USP-São Paulo em 1976; o segundo na UFSC, organizado por Walter Celso de Lima em 1978; o terceiro CBA ocorreu no Rio de Janeiro, organizado por Liu Hsu da UFRJ, em 1980; o quarto CBA ocorreu em Campinas, organizado por Celso Bottura da UNICAMP, em 1982.

- No primeiro CBA não conseguimos produzir os Anais, que só foram produzidos e entregues juntamente com os do segundo CBA, quando do início do congresso. Hoje as coisa mudaram, como já comentei. Fazer os anais com base em artigos revisados pelos pares sempre foi um ponto importante e trabalhoso na organização de um congresso de nível adequado e o CBA manteve tal tradição com muito respeito e empenho de suas comissões organizadoras.

Para a segunda diretoria da SBA-1977-1979 foi eleito presidente Antonio Salles de Campos Filho, do ITA e Celso Bottura continuou como vice-presidente. Em 1978, o presidente renunciou e Celso Bottura assumiu a presidência da segunda diretoria da SBA. Para a terceira diretoria (1979-1981) e excepcionalmente para a quarta diretoria da SBA, Walter Celso de Lima da UFSC foi eleito presidente. Para a quinta diretoria da SBA, Celso Bottura da UNICAMP foi eleito presidente.

Em 1984, a SBA juntamente com a Associacion Argentina de Control AutomáticoAADECA e a Associacion Chilena de Control Automático-ACCA organizaram o Primeiro Congresso Latino-Americano de Automática, em Campina Grande-PB, Brasil sob a liderança de Edson Roberto Cabral da Silva. Ele foi realizado conjuntamente com o quinto CBA. Desde então, a cada 2 anos ocorrem os Congressos Latino-Americanos de Automática; o segundo em Buenos Aires, Argentina-1986; o terceiro em Santiago,Chile-1988. O quarto em Puebla, México, organizado pela AMCA-Associacion de México de Control Automático em 1990. O quinto em Havana, Cuba, organizado pela CONAI-Comission Nacional de Automatizacion Industrial em 1992.

- A SBA já organizou três Congressos Latino-Americanos de Automática no Brasil: - Campina Grande, PB, 1984; Rio de Janeiro, RJ,1994 e Salvador, BA, 2006. - Se para a América Latina, a primeira Latin-American Control Conference ocorreu em 1984; se para a Europa, a primeira European Control Conference ocorreu em 1991 e para a Ásia, a primeira Asian Control Conference ocorreu em 1994, falta ainda ocorrer para o continente América, a primeira Pan-American Control Conference [32].

O primeiro evento internacional IFAC organizado pela SBA foi o "IFAC Symposium of Planning and Operation of Electric Energy Systems", no Rio de Janeiro em 1985, organizado por Acher Mossé, do CEPEL-Centro de Pesquisas de Energia Elétrica da Eletrobrás.

Para a sexta diretoria da SBA-1985-1987, Atair Rios Neto do INPE, foi eleito presidente. Ele criou em 1987 a revista SBA-Controle e Automação. Os editores da revista Controle e Automação foram: Wagner Caradori do Amaral, UNICAMP, 
1987-1989, José Cláudio Geromel, UNICAMP, 1990-1991, Rafael dos Santos Mendes, UNICAMP,1992-1995, Oswaldo Luiz do Valle Costa, USP, 1996-1999, João Bosco Ribeiro do Val, UNICAMP, 2000-2004, Ricardo Ribeiro Gudwin, UNICAMP, 2004-2008. Luiz Antonio Aguirre, UFMG, foi o editor de 2009 a 2012 e passou de 4 para 6 o número anual de revistas publicadas.

Em 1993, durante a presidência de Pedro Guimarães Ferreira, da PUC-RJ, a SBA criou, por iniciativa de Márcio Rilo, da USP, que viria a ser o décimo primeiro presidente da SBA, o SBAI-Simpósio Brasileiro de Automação Inteligente, um congresso bianual. O primeiro SBAI ocorreu em Rio Claro, SP em 1993; o décimo foi em São João Del Rei, MG, em setembro de 2011.

Recentemente, em 2008, por iniciativa, patrocínio e organização da SBA, os três primeiros volumes da Enciclopédia de Automática, foram publicados pela editora Blucher de São Paulo [1]. Ela foi escrita por quase uma centena de membros muito ativos da SBA, de 27 diferentes instituições brasileiras, tendo o Professor Luis Antonio Aguirre, da UFMG, como Editor.

\section{Reflexões e Conclusões}

Quando preparava este trabalho, recebi, como regularmente recebo, a revista "Options, Summer 2011"do "IIASA-International Institute for Applied Systems Analysis". Fiquei muito feliz com a seguinte notícia:

- "IIASA National Member Organizations-NMOs: Brazil and Malaysia join IIASA"Nela fiquei sabendo, com enorme satisfação, que "the Center for Strategic Studies and Management in Science, Technology and Innovation(CGEECentro de Gestão e Estudos Estratégicos) of Brazil and the Academy of Sciences of Malaysia", a partir de fevereiro de 2011 são NMOs do IIASA[22].

Este fato levou-me de volta a 1974, quando como chefe do Departamento de Automação da UNICAMP, visitei o IIASA, com grandes sonhos e entusiasmo. Tal excelente notícia provocou-me memórias e reflexões que tem muito a ver com dinâmica, controle e matemática aplicada:

- O IIASA era criação recente durante a Guerra Fria entre Estados Unidos e seus aliados e a então União Soviética e seus aliados. Como a Austria estava estrategicamente situada, foi escolhida para ter, dentre outras instituições, o IIASA, um centro de pesquisa de alto nível com cientistas dos 2 lados da então "cortina de ferro".

- Na minha visita ao IIASA em 1974 tive reuniões com pesquisadores em Controle de Sistemas Dinâmicos,que lá trabalhavam, em particular com o americano Irving Lefkowits, Professor da Case-Western Reserve University, USA, e com o russo A. M. Letov[24], grande autoridade em controle de sistemas não lineares e muito famoso na época. Também reuni-me com o americano Howard Raiffa, que dirigia o IIASA, era da Harvard University, USA , e tinha escrito um importante livro sobre teoria dos jogos[26], assunto em que eu viria a orientar 2 teses de doutorado com resultados muito bons e úteis $[15,16]$, 
em particular para o controle com múltiplos agentes, uma área cada vez mais importante e interessante.

- De volta ao Brasil, em 1974, como chefe do Departamento de Automação provocamos uma primeira grande reestruturação nos cursos de pós-graduação na área de Controle e Automação da engenharia elétrica da UNICAMP [33]. Neste trabalho foram fundamentais as participações dos Professores Hermano Tavares, que posteriormente seria Presidente da SBA, Reitor da UNICAMP, Reitor Fundador da UFABC-Universidade Federal do ABC, Presidente da Associação dos Docentes da UNICAMP-ADUNICAMP, e que enfatizou o desenvolvimento de ensino e pesquisa em Programação Matemática, particularmente em Programação Não Linear e Linear; Yaro Burian Junior em Sistemas Não Lineares, Máquinas Elétricas e Teoria de Circuitos; Luiz Gimeno Latre, Manuel de Jesus Mendes, Marcio Luiz de Andrade Netto em Controle Ótimo, Controle Estocástico, e Computação; o Professor Mario Jino em Engenharia de Software; o Professor Gerard Authié em Programação Dinâmica e Programação Matemática de Grande Porte; o Professor Celso Bottura em Teoria de Sistemas Lineares e Não Lineares, Controle de Acionamento, Controle Multivariável e em Controle e Otimização de Sistemas Dinâmicos de Grande Porte.

- Estas atividades tinham correlação profunda com as atividades do IIASA, que voltei a visitar em 1996. Sempre desejei que o Brasil fosse MEMBRO DO IIASA e sonhei em lá passar um período como"Visiting Scholar", similarmente ao que me ocorreu ao ser um "Fulbright Visiting Scholar"do Departamento de Economia da UCLA-University of California-Los Angeles, USA, em 19841985 .

- Estou muito contente com a filiação do Brasil ao IIASA e espero que muitos dos integrantes da nossa comunidade brasileira de Dinâmica e Controle se beneficiem direta e/ou indiretamente desta importante e acertada decisão brasileira.

A partir do final da década de 1980, começou no Departamento de Engenharia Mecânica da Escola Politécnica da USP um curso com habilitação em Automação e Sistemas, que foi de fato o primeiro curso denominado de Mecatrônica no Brasil. Em 2000 este curso passou a ser denominado Engenharia Mecatrônica, após ampla reformulação curricular.Desde então, este tipo de curso de Controle e Automação se difundiu pelo país, bem como a palavra Mecatrônica[1]. Eu penso que a palavra Automática é mais abrangente e exprime melhor as atividades em Controle e Automação. Em 1988, o Departamento de Engenharia Mecânica da Faculdade de Engenharia de Campinas tornou-se a Faculdade de Engenharia Mecânica-FEM da UNICAMP. A partir da década de 1990, cursos de controle para os alunos de engenharia mecânica da UNICAMP passaram a ser oferecidos pelo Prof. Eurípedes Guilherme de Oliveira Nóbrega, da FEM, e engenheiro eletrônico formado pelo ITA. No final da década de 1990, liderado pelos Profs, Douglas Eduardo Zampieri, João Maurício Rosário, e Eurípedes Nóbrega, deu-se a criação na FEM, do curso de Engenharia de Controle e Automação da UNICAMP. 
As atividades na área de Controle e Automação cresceram muito no mundo, e em particular em nosso país. Há, e haverá ainda mais, cursos nos mais variados níveis e especialidades. Eles são tradicionais hoje nas engenharias elétrica, mecânica, aeronáutica, química, mecatrônica, controle e automação, tanto na graduação como na pós-graduação. Em cursos técnicos também ocorrem importantes e essenciais atividades para esta área. Em cursos de economia, controle ótimo e estimação de estado têm e terão inserção cada vez maior.

- O controle de sistemas quânticos, a geometria diferencial e o controle e a estimação inteligentes de sistemas não lineares, são temas cada vez mais importantes.

- Automática e Matemática são atividades distintas, mas que têm interseç̧ões importantes; assim como Automática e Física, Controle e Economia, Controle e Finanças, Controle e Biologia, ...

- Dentro do Departamento de Automação da UNICAMP, que existiu como tal até 1976, começaram as atividades de Engenharia Biomédica da UNICAMP, com os professores Maria Adélia Collier Farias, que me sucedeu na chefia do Departamento de Automação, Wang Binseng e José Wilson Magalhães Bassani, orientados da prof. Maria Adélia. Para que isso ocorresse as interações com os Professores Renato Terzi e Nubor Facure, da Faculdade de Ciências Medicas da UNICAMP, foram essenciais, bem como a liderança do Prof. Manoel Sobral Junior. O sucesso destas atividades na UNICAMP nos dá muita satisfação e boas lembranças.

Em 1976, os Departamentos de Automação, de Eletrônica e Comunicações e de Eletrotécnica da Faculdade de Engenharia de Campinas foram reunidos em um grande Departamento de Engenharia Elétrica com mais de 100 professores em tempo integral, a maioria com doutorado; dada a sua dimensão e qualidade, tornou-se em 1986, a Faculdade de Engenharia Elétrica-FEE da UNICAMP, cujo primeiro diretor foi o Professor Hermano Tavares. Nesta reformulação criamos na FEE-UNICAMP, o Departamento de Máquinas, Componentes e Sistemas Inteligentes-DMCSI, que chefiei nos primeiros sete anos. Nele criamos o Laboratório de Controle e Sistemas Inteligentes-LCSI, que dirigi até aposentar-me em 2003. Posteriormente, a FEE tornou-se a Faculdade de Engenharia Elétrica e de Computação-FEEC da UNICAMP, na década de 1990. Penso que a dinâmica deste sistema, que muito me interessa, é da maior importância para o Brasil, em particular para o seu ENSINO SUPERIOR. De certa forma, o que estava ficando difícil de realizar no ITA, sobretudo a partir da sua crise de 1965, tornou-se viável com a criação e o desenvolvimento da UNICAMP, e a Reforma Universitária que se seguiu.

A inter-disciplinaridade de Controle de Sistemas Dinâmicos é uma de suas mais importantes características. Graças a ela realizamos:

- Pesquisas e desenvolvimentos que nos permitiram contribuir para as áreas de Controle de Acionamento, Eletrônica de Potência e Eletroquímica, tão essenciais e construir Veículo Elétrico que percorreu o Campus da UNICAMP 
em 1984[2, 9]. - Idem em Programação Matemática, Computação, Inteligência Artificial, Pesquisa Operacional, Teoria de Jogos Dinâmicos, Matemática Aplicada e Computacional, Modelagem Computacional de Dados e Dinâmica e Controle de uma forma geral[ 7,8$]$.

ESPERAMOS QUE ISTO CONTINUE EM NOSSA UNIVERSIDADE, CONGRESSOS E DEMAIS ATIVIDADES EM NOSSA ÁREA, em particular na Faculdade de Engenharia Elétrica e de Computação da UNICAMP, onde estou há mais de 44 anos, atualmente como Professor Colaborador Voluntário.

Com a valorização da educação, da tecnologia e da ciência em nosso país, e com o amadurecimento dos nossos pesquisadores, pergunto nestas reflexões se não seria oportuno:

- Estudar, Compreender e Aperfeiçoar o Papel da Academia Brasileira de CiênciasABC? Idem, de Sociedades Científicas Brasileiras?

Bem como criar:

- A Academia Brasileira de Ciências da Engenharia-ABCDE?

- A Financiadora Paulista de Projetos-FPP?

\begin{abstract}
Graduate education started in Brazil in 1961. In these 50 years the range and importance of this initiative and its influence on the Brazilian Universities, both for research and for teaching, are enormous. The Doctorate as it occurs nowadays in Brazil started in 1969, almost as an artisan's work, due to the lack of experience in doing research in our country at that time, and gradually started to grow. A highly successful maturing and growing process occurred in the last 20 years. We may say that nowadays we have mature researchers and Graduate Programs in the most varied areas and Brazilian Universities, in spite of our science and our engineering are still far away from the ones the best world centers do. In parallel and because of such a process, various scientific societies and/or conferences were being created and consolidated. In this paper I present some memories and reflections about this process emphasizing my working area as Professor and Researcher: Control of Dynamic Systems and in my life experience.
\end{abstract}

Keywords. Control, dynamic systems, history.

\title{
Referências
}

[1] L.A. Aguirre (Ed.), "Enciclopédia de Automática", Vols. 1,2,3, Editora Blucher, 2007.

[2] G. Barreto, M.G. Oliveira, C.P. Bottura, "Desempenho de baterias chumboácido: estudo experimental e modelamento do processo de descarga", $3^{\circ}$ Congresso Brasileiro de Energia, 1984.

[3] C.P. Bottura, "Simulador para Estudo da Estabilidade Dinâmica Longitudinal de Aviões Utilizando Computador Analógico", T.I., ITA, São José dos Campos, SP, 1962. 
[4] C.P. Bottura, "Contribuição ao Estudo do Controle de Torque de Máquina Série por Modulação em Largura de Pulso", Tese de Doutorado, UNICAMP, Campinas, SP, 1973.

[5] C.P. Bottura, "Análise Linear de Sistemas", Editora Guanabara Dois, Rio de Janeiro, RJ, 1982.

[6] C.P. Bottura,"Princípios de Controle e Servomecanismos", Editora Guanabara Dois, Rio de Janeiro, RJ, 1982.

[7] C.P. Bottura, "Memorial para Concurso Público para Professor Titular na Área de Sistemas de Controle", UNICAMP, Campinas, SP, 1999.

[8] C.P. Bottura, Curriculo Lattes, (n.d.)

[9] C.P. Bottura, G. Barreto, "Veículos Elétricos", Editora da UNICAMP, Campinas, SP, 1989.

[10] C.P. Bottura, C.E. Pereira, "Points in the History of the Brazilian Society of Automatics (Sociedade Brasileira de Automática-SBA)", Controle e Automação, Vol. 22, No. 1 (2011), 1-8.

[11] Y. Burian Jr, C.P. Bottura, Controle por comutação de motor série, Anais do $2^{\circ}$ Congresso Brasileiro de Engenharia Mecânica, Vol. C, 1973, 621-636.

[12] P.B.L. Castrucci, "Controle Automático: Teoria e Projeto", Editora Edgar Blucher, São Paulo, SP, 1969.

[13] M.A.E. Cecchini, "O ITA Que Eu Conheci", Edição do Autor, São José dos Campos, SP, 2011.

[14] P. Colaneri, J.C. Geromel, A. Locatelli, "Control Theory and Design: An RH2 and RHinfinite Viewpoint", Academic Press, 1997.

[15] E.A. Costa, "Matriz de Jogos Estratégicos- Novo Modelo para Representação e Estudo de Conflito de Interesses", Tese de Doutorado, UNICAMP, Campinas, SP, 2008.

[16] J.T. Costa Filho, "Proposta Para Computação Assíncrona Paralela e Distribuída de Estruturas Especiais de Jogos Dinâmicos", Tese de Doutorado, UNICAMP, Campinas, SP, 1992.

[17] J.C. Geromel, R.H. Korogui, "Controle Linear de Sistemas Dinâmicos", Editora Blucher, São Paulo, SP, 2011.

[18] J.C. Geromel, A.G.B. Palhares, "Análise Linear de Sistemas Dinâmicos", segunda edição, Editora Blucher, São Paulo, SP, 2011.

[19] J. Gertler (Ed.), "Historic Control Textbooks", International Federation of Automatic Control/Elsevier, 2006.

[20] E. Gomes, "O Mandarim", Editora da UNICAMP, Campinas, SP, 2006. 
[21] L.T.C.T. Gomes, "Estudo da Estabilidade Dinâmica do Avião IPD-PAR/6504 e Simulação no Computador Analógico", T.I., ITA, São José dos Campos, 1968.

[22] IIASA Options, Summer 2011, 4.

[23] R.J. Jacquot, "Modern Digital Control Systems", 2nd Edition, Marcel Dekker, New York, 1994.

[24] A.M. Letov, "Stability of Nonlinear Control Systems", Princeton University Press, Princeton, 1961.

[25] O.L. Linhares, "Sobre a Racionalização de Dois Algorítmos Numéricos", Tese de Doutorado, EESC-USP, São Carlos, SP, 1968.

[26] R.D. Luce, H. Raiffa, "Games and Decisions", Wiley, New York, 1957.

[27] F. Morais, "Montenegro", Editora Planeta do Brasil Ltda., São Paulo, SP, 2006.

[28] A.G.B. Palhares, "Controle por Realimentação de Estado e Modulação em Largura de Pulso de um Servoposicionador Eletrohidráulico: Análise das Oscilações Instáveis", Tese de Doutorado, UNICAMP, Campinas, SP, 1980.

[29] C.E. Pereira, C.P. Bottura, The Brazilian Society of AutomaticsSBA("Sociedade Brasileira de Automática-SBA"). The 2009 Conference on the History of Technical Societies, Philadelphia, Pennsylvania, USA, 2009.

[30] O. Silva, D. Fischetti, "Casimiro Montenegro Filho", Bizz Editorial, São Paulo, SP, 2006.

[31] H.M.F.Tavares, Coordenador, "Frenagem e Tração do Material Rodante", $1^{\circ}$ relatório do Convênio Metro-UNICAMP PS-2/160, Faculdade de Engenharia de Campinas, UNICAMP, Campinas, SP, 1974.

[32] M. Thoma, R.J. Patton (Eds.), "Proceedings of the European Science Foundation Workshop: Control of Complex Systems", La Sapienza, Rome, 1995.

[33] UNICAMP, "Catálogo de Pós-Graduação 1976", 1976. 